Meta

Journal des traducteurs

Translators' Journal

\title{
L'expressivité : essai de définition
}

\section{Yvonne Saint-Pierre Farina}

Volume 18, numéro 3, septembre 1973

URI : https://id.erudit.org/iderudit/002731ar

DOI : https://doi.org/10.7202/002731ar

Aller au sommaire du numéro

Éditeur(s)

Les Presses de l'Université de Montréal

ISSN

0026-0452 (imprimé)

1492-1421 (numérique)

Découvrir la revue

Citer cet article

Saint-Pierre Farina, Y. (1973). L'expressivité : essai de définition. Meta, 18(3),

315-327. https://doi.org/10.7202/002731ar d'utilisation que vous pouvez consulter en ligne.

https://apropos.erudit.org/fr/usagers/politique-dutilisation/ 


\section{L'expressivité : essai de définition}

\section{LES ÉLÉMENTS}

On sait que la stylistique genevoise étudie les moyens expressifs mis à la disposition du groupe linguistique par le legs collectif. Il s'ensuit que définir l'expressivité revient à définir la stylistique telle que nous l'entendons. L'on comprend toute l'importance de circonscrire ce terme.

L'expressivité se mesure par la proportion d'affectivité et de concret dégagée par le mot ou l'expression. Plus le terme est affectif et concret, plus il est expressif ; moins il est affectif et concret, moins il est expressif. L'expressivité se constitue donc par deux pôles : l'affectivité s'adressant aux émotions et le concret appelant une réaction des sens et de l'imagination. Ces deux pôles peuvent se fusionner ou se séparer ; augmenter à l'infini ou diminuer jusqu'à la disparition ; ils peuvent manifester ou inspirer soit un attrait, soit une répugnance vis-à-vis de la réalité signifiéé; ils peuvent nous en faire saisir les éléments tactiles, auditifs, visuels, etc., dans leurs détails ou tout simplement laisser ceux-ci dans l'ombre pour faire ressortir la désidérabilité ou la non-désidérabilité de ce dont il s'agit. En d'autres termes, l'expressivité peut jouer sur toute la gamme des émotions et des sens.

\section{L'affectivité}

Celle-ci est constituée par la présence d'une composante sémantique comportant une intensité, c'est-à-dire le degré ou la quantité expressive (énorme, intensif de gros; minuscule, intensif de petit) ; une qualité (favorable ou défavorable : sublime, méprisable) ; ou l'évocation d'un milieu, d'une situation spéciale, causant ainsi une réaction émotive en établissant une relation temporelle (chef, terme d'un autre siècle, employé dans une conversation plutôt que tête, terme $\mathrm{du} \mathrm{xx}^{\mathrm{e}}$ siècle), une relation géographique (vodka, terme russe utilisé dans les pays francophones), une relation sociale (caboche, terme argotique utilisé pour tête par un professeur dans une conférence universitaire), une relation traditionnelle (bourreau, terme conceptuel traditionnellement lié à une situation émotive), ou une 
relation linguistique où l'esprit doit faire un circuit sémantique pour interpréter la situation comme dans la métaphore et la comparaison (cet homme est un lion). L'intensité, la qualité ou la relation, séparément ou toutes ensemble, peuvent doter le terme d'une charge émotive capable de manifester ou de modifier, selon le cas, des dispositions positives ou négatives. Lorsque celles-ci ont pour but principal de révéler ou d'influencer les réactions émotives, elles sont affectives et les mots à dominance affective sont des mots émotifs.

\section{Le concret}

Le concret est une réalité percevable par les sens : la vue, l'ouïe, le toucher, le goût, l'odorat, la tension musculaire, et ainsi de suite. C'est à la psychologie qu'il faut s'adresser pour découvrir une liste plus complète des sens.

Les mots reflètent l'acuité de perception sensible. D'un côté, ils représentent des réalités saisissables par l'intelligence seulement (la philosophie, l'unité, un groupement) ; d'autre part, ils signifient une réalité percevable par les sens, mais d'une façon incolore, générale, et conceptualisante (lit, pupitre, marcher). Ces termes généraux s'adressent surtout à l'intelligence et leur expressivité est ou bien réduite à un minimum, ou bien inexistante. La langue est cependant pleine de termes qui précisent d'une façon souvent très pittoresque les perceptions des sens (fourmiller, gambader, glouglou, crisp, fuzz, zoom). Cette précision domine et noie, pour ainsi dire, l'élément conceptuel contenu dans le terme. C'est de ce concret détaillé qu'il s'agit. Ce sont là des mots pleins d'expressivité. Ce sont des mots-images : des mots qui font image.

\section{RAPPORTS ENTRE LES ÉLÉMENTS EXPRESSIFS}

Un graphique montrera les rapports entre les éléments expressifs qui peuvent se trouver ensemble dans le même mot ou s'isoler, selon le cas (cf. p. 317).

\section{L'intensité}

Ayant ainsi replacé les éléments par rapport à l'ensemble, l'on peut se mettre à examiner ceux-ci.

La première modalité affective des termes expressifs est l'intensité. Elle exprime le degré élevé auquel sont portés telle propriété, telle action, tel mouvement, etc. Elle constitue une rubrique en soi, puisque l'intensité peut sous-tendre la qualité, la relation et le concret pittoresque sans perdre pour autant la possibilité de les dominer. Le péjoratif et le mélioratif, sans pourtant s'effacer, laissent à l'intensité le soin de remporter notre adhésion ou d'obtenir notre désapprobation. Voici des exemples où la dominance est intensive :

... que les Sorcières thessaliennes contraignent durement à danser sur l'herbe terrifiée 1 !

"And yet", said the Doctor, "I am filled with a wholly delightful exhilaration 2. " It would be a great comfort to express the immensity of his contempt 3 .

Le Panturle est un homme énorme 4 .

1. Charles Baudelaire, le Spleen de Paris, Paris, Le Livre de Poche, 1966, p. 111.

2. Evelyn Waugh, "Decline and Fall », in Seven Great British Short Novels, New York, Berklay Medallion, 1968, p. 312.

3. Somerset W. Maughan, Of Human Bondage, New York, 1968, p. 217.

4. Jean Giono, Regain, Paris, Le Livre de Poche, 1965, p. 16. 


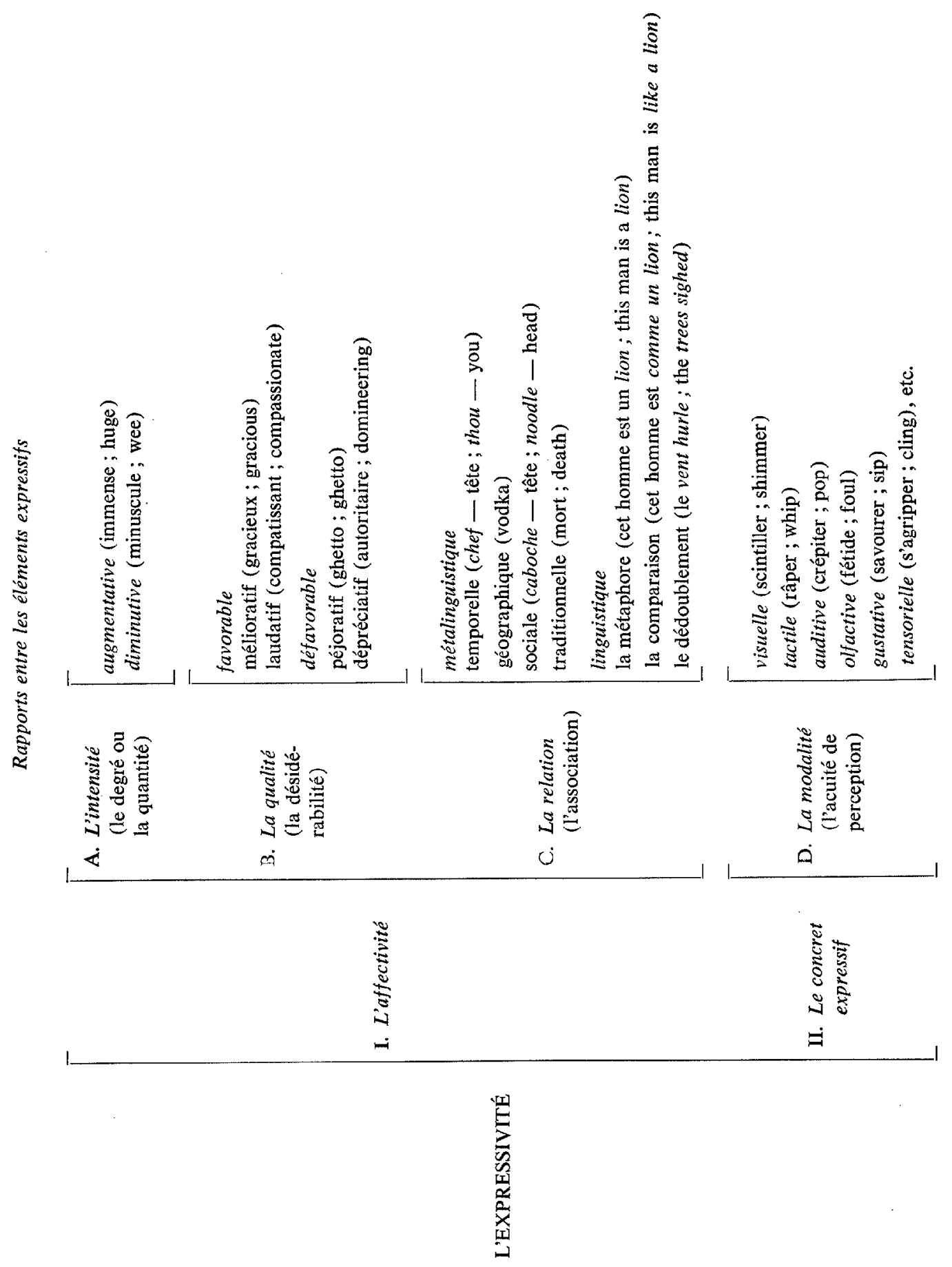


Ces exemples expriment un degré élevé de peur (terrifiée), d'animation (exhilaration), de plénitude (wholly), de grandeur (immensity), de grosseur (énorme), de désapprobation (contempt), et d'acceptation (delightful). La sensibilité, surprise ou éprise par l'intensité, colore le concept et le représente à travers un prisme émotif. Le degré forme une composante sémantique du terme, comme l'on s'en apercevra si l'on cherche la définition de énorme pour ce contexte : hors de l'ordinaire par sa grosseur. Grosseur est l'identification neutre du concept, tandis que hors de l'ordinaire explicite l'intensité. Certains termes ont donc pour rôle spécial de porter l'attention sur le degré où se situe la réalité concrète ou affective, mais ce degré émeut parce qu'il dépasse l'attente.

L'intensité est ambivalente. Elle peut être ou augmentative ou diminutive. Elle est augmentative si elle dépasse les bornes dans une espèce de croissance dynamique; elle est diminutive si elle s'achemine vers une décroissance. Dans un cas, elle oriente l'affectivité vers l'infiniment grand; dans l'autre, elle va vers l'infiniment petit. Mais dans les deux cas la réalité sort des règles et ainsi elle émeut. Voici des exemples où l'intensité est augmentative :

Buenos Aires, déjà, emplissait l'horizon de son feu rose, et bientôt luirait de toutes ses pierres, ainsi qu'un trésor fabuleux 5 .

Robert Jordan explained the process of homesteading... "That is magnificent », Primitivo said ${ }^{6}$.

... remember that butter has to do for three loaves. Spread it thoroughly, but don't waste it... ${ }^{\top}$

On the fourth side was an iron railing through which you saw a vast lawn 8 .

He swept out of the room with giant strides... 9

Il lui donne un coup de poing dans les côtes. Étonnée, Caroline esquive un autre coup en creusant les reins 10 .

Dans tous ces exemples, le rôle du terme est de transformer le concept en une expression affective : rare (fabuleux), acceptable (magnificent), bien (thoroughly), grand (vast, giant), sortir (swept out of), surprise (étonnée). Le bien, le rare, l'acceptable, le grand, la surprise, l'action de sortir dépassent les bornes de l'attente et c'est précisément ce mouvement croissant que ressent la sensibilité et qu'elle exprime en forgeant des mots au même concept mais à modalité affective différente.

L'on peut sortir de l'ordinaire dans un sens contraire, c'est-à-dire que l'on peut dépasser l'attente par la diminution et que cette diminution marque une sorte de transition dynamique vers l'infiniment petit :

Il donne des détails minutieux sur ses fonctions... 11

De lourdes pendeloques gazouillent secrètement à ses mignonnes oreilles 12 .

C'est presque au milieu d'un tas de décombres que Gédémus a découvert une grangette encore tiède 13 .

5. Antoine de Saint-Exupéry, Vol de nuit, Paris, 1965, p. 174.

6. Ernest Hemingway, For Whom the Bells Toll, New York, Scribners, 1940, p. 207.

7. Evelyn Waugh, op. cit., p. 313

8. Somerset W. Maughan, op. cit., p. 18.

9. Somerset W. Maughan, op. cit., p. 18.

10. Jean Giono, op. cit., p. 82.

11. Colette, l'Ingénue libertine, Paris, Le Livre de Poche, 1962, p. 170.

12. Charles Baudelaire, op. cit., p. 74.

13. Jean Giono, op. cit., p. 62. 
Some tiny scrap of life, like a grain of smut, went racing across the page in front of him... ${ }^{14}$

Excessif (minutieux), petit (mignonnes), grange (grangette), small (tiny) sont des concepts dont s'empare l'affectivité pour les orienter vers l'atome. Les mots utilisés dans ces textes sont aussi intenses dans leur rétrécissement que dans les exemples précédents, mais le dynamisme affectif de ceux-là 's'orientait dans le sens contraire. L'intensité est donc passible d'une expression affective à deux orientations, les deux visant l'infini : l'un grand, l'autre petit. Les bornes sont floues, leur potentiel n'est limité que par l'imagination et les puissances affectives de l'individu.

\section{La qualité}

Le mouvement vers l'infini, déjà noté, peut ne pas toujours être désirable. L'individu est amené à considérer certaines réalités comme des menaces à son bien-être, tandis que d'autres semblent le favoriser. La sensibilité juge telle chose favorable et telle autre défavorable. Elle est bien disposée vers telle réalité ou bien elle ne l'est pas. Si elle en ressent l'attrait, on dit que le mot est mélioratif ou laudatif. Ce dernier se rapporte au moral seulement, le premier à toute autre réalité-objet. Si le mouvement affectif est contraire, l'affectivité s'exprime par un mot péjoratif ou dépréciatif. Si le mot est péjoratif, il s'occupe de tout ce qui est en dehors du domaine moral ; s'il est dépréciatif, il s'occupe du côté moral. La ligne de démarcation entre mélioratif et laudatif, péjoratif et dépréciatif hésite parfois, étant donné que les liens sont étroits entre le moral et le physique, le moral et le social, pour ne fournir que deux exemples. En principe, il est convenable de retenir cette distinction en raison même des effets spéciaux que dégagent de tels termes en dehors de leur propre domaine (ex. : le soleil nous boude). $\mathrm{Ce}$ qu'il importe de noter par-dessus tout est l'ambivalence, c'est-à-dire la double orientation du jugement de valeur affectif. Voici quelques exemples :

Mélioratif :

Antoine l'admire et jubile, à demi caché derrière son Figaro... ${ }^{15}$

« He's just crazy to meet the aristocracy, aren't you, my sweet 16 ? »

... he experienced a charming sense of possession in installing her in these lodgings... ${ }^{17}$

J'aime les nuages, les merveilleux núages 18 !

Laudatif :

... et plus l'âme est ambitieuse et délicate, plus les rêves l'éloignent du possible ${ }^{19}$.

Il en tâte, entre ses doigts, toute la bonne volonté 20.

"I'm an optimist ", Mr. Tench said 21.

14. Graham Greene, The Power and the Glory, New York, Bantam Books, 1940, p. 131.

15. Colette, op. cit., p. 21.

16. Evelyn Waugh, op. cit., p. 325 .

17. Somerset W. Maughan, op. cit., p. 165.

18. Charles Baudelaire, op. cit., p. 15.

19. Charles Baudelaire, op. cit., p. 25.

20. Jean Giono, op. cit., p. 184.

21. Graham Greene, op. cit., p. 11. 
Péjoratif :

She looked grotesque [...] she had never seemed so unattractive [...] he could not forget his dismay when she turned round and he saw her in her camisole and the short petticoat [...] he did not see how she could be less than forty. It made the affair ridiculous. She was plain [...] in those frocks which were too showy for her position and too young for her years... 22

... un sol aussi désolé que le ciel ${ }^{23}$.

Dépréciatif :

... est-ce bête? n'est-ce pas un peu vulgaire? immoral ? grossier 24 ?

... un âne trottait vivement, harcelé par un malotru armé d'un fouet... 25

You naughty boy, Miss Watkin will be cross with you 26 .

Maudites soient vos ambitions... 27

Qu'on note, sous la rubrique «laudatif », le curieux exemple du mot ambitieuse, et qu'on le compare avec ambitions sous la rubrique «dépréciatif ». Ce terme, comme d'ailleurs son équivalent anglais, peut, selon le contexte, prendre deux orientations affectives, l'une favorable, l'autre défavorable. Le même terme est utilisé dans les deux cas : c'est la situation qui en détermine l'acheminement. Ce sont des termes affectifs à qualité ambivalente.

\section{La relation}

Cette ambivalence est caractéristique de toute l'affectivité. L'intensité oriente vers l'infini : l'infiniment grand et l'infiniment petit. La qualité tourne autour des dispositions négatives ou positives. La relation constate l'assimilation ${ }^{28}$ ou l'aliénation par rapport au milieu connu de l'individu ou du groupement linguistique. La relation est, ni plus ni moins, ce rapport qui existe entre deux temps, deux milieux, deux situations, deux objets, représentés par des mots dont l'un tend à s'assimiler à la situation de l'autre (la métaphore), ou dont l'un refuse de s'assimiler à la situation de l'autre : résiste, hésite. C'est le cas de la relation temporelle, géographique, sociale. Dans le cas de la relation traditionnelle, une situation intellectuelle s'assimile à une situation affective tout en retenant le même mot pour exprimer l'une et l'autre. Le rapport peut doter le mot aliéné ou assimilé d'une expressivité que l'unité sémantique est souvent incapable de porter en soi. Il s'ensuit que, d'ordinaire, l'affectivité résultant d'une relation est accidentelle, c'est-à-dire que le mot ne prend une valeur affective que dans le contexte ou dans une situation temporaire s'il ne contient pas déjà sa propre dominance qualitative et intensive.

1. La relation peut être métalinguistique plutôt que linguistique, quand elle tient son origine en dehors de la langue, à savoir dans le temps, dans le milieu géographique et social, ou dans la tradition.

22. Somerset W. Maughan, op. cit., p. 70.

23. Charles Baudelaire, op. cit., p. 24.

24. Nathalie Sarraute, le Planétarium, Paris, Le Livre de Poche, 1968, p. 20.

25. Charles Baudelaire, op. cit., p. 19.

26. Somerset W. Maughan, op. cit., p. 32.

27. Charles Baudelaire, op. cit., p. 129.

28. L'assimilation est la conversion d'un mot en un terme employé couramment par tout le groupe linguistique, ce qui, avec le temps, en fait oublier l'origine et lui enlève l'effet affectif dû à cette origine. 
a) La relation temporelle : le mot provient d'un autre siècle et n'est pas assimilé par le groupement linguistique d'aujourd'hui : il détonne et surprend l'affectivité. Souvent la réalité qu'il représente est disparue. Le mot tend alors à prendre un halo exotique ou mystérieux ou simplement «différent». Parfois l'on crée le mot de toutes pièces en raison d'une situation nouvelle et il n'a pas encore eu le temps de s'assimiler au bagage linguistique du groupement. Le mot sent « étranger ». On reconnaît l'archaïsme et le néologisme. Si l'on disait aujourd'hui : "couvre-toi le chef » au lieu de «mets ton chapeau» ou «couvre-toi la tête », on serait sûr d'une réaction comme c'est le cas dans les exemples suivants :

"Where is the rifle pointed? " asked Anselm into the dark. "At thee ", the man said 29. She was - do you know the Newspeak word goodthinkful? Meaning naturally orthodox, incapable of thinking a bad thought 30 ?

- Allons, dit Bianchon, il parlait de ses filles, après lesquelles il crie comme un homme sur le pal crie, dit-on, après l'eau 31 .

Le pal est une réalité sortie des mœurs, thee ne s'emploie plus en conversation ordinaire, Newspeak et goodthinkful sont de pures inventions dont les lendemains sont moins que sûrs. De tels termes nous parlent de ce que l'on ne connaît pas ou de ce que notre époque n'assimile pas. Ils comportent un élément de mystère, de nouveauté, de jamais-vécu, même s'ils proviennent du passé, même si l'on en comprend peu les éléments concrets ou les nuances affectives contenues dans leur signification. On ne peut pas ne pas réagir.

b) La relation géographique : le mot est bien de notre siècle mais il provient d'une autre région, d'un autre pays, et n'est pas complètement assimilé à la culture du groupement linguistique qui l'adopte. Il dégage des nuances souvent exotiques, quelquefois il reflète un certain cosmopolitisme. Il surprend plus ou moins et réagit sur la sensibilité dans la même mesure où il est reconnaissable comme emprunt :

Maintenant, la Mamèche est là, seule, devant la Vierge qui rit sous la crème du lait. - Bellissima!

Elle a un élan de ses grands bras noirs.

- Mia Bella, celle que j'aime plus que tout, viens que je t'essuie 32 .

Les cultures italienne et française se rencontrent dans la même phrase, mais le mariage n'est pas fait.

c) La relation sociale : le mot provient d'un autre niveau social dans le même pays et le même siècle :

With or without her nigger, Mrs. Beste-Chetwynde was a woman of vital importance [...] "Niggers are alright", said Philbrick. "Where I draw a line is a Chink, nasty inhuman beings... 33 ,

29. Ernest Hemingway, op. cit., p. 44.

30. George Orwell, 1984, New York, The American Library, 1961, p. 110.

31. Honoré de Balzac, le Père Goriot, Paris, Le Livre de Poche, 1965, p. 420.

32. Jean Giono, op. cit., p. 34.

33. Evelyn Waugh, op. cit., p. 324ss. 
The lrish heart where'er it be

Is bowed with woe - for he is gone

Who would have wrought her destiny 34 .

On reconnaît le niveau de langue poétique. Ces termes frappent parce qu'ils sortent de l'usage commun et ne s'utilisent guère que dans certains milieux spéciaux.

- Je ramène la brebis perdue, dis-je, avec le plus de solennité que je pus $3 \bar{n}$.

Ici l'allusion biblique relève le ton et nous transporte hors du quotidien.

La relation sociale tient compte de la langue employée par les divers milieux sociaux - littéraire, poétique, familière, patoise, dialectale et ainsi de suite. Ces divers parlers, sortis de leur milieu, apportent avec eux la saveur de leur tonalité particulière, en plus des éléments concrets et affectifs déjà contenus dans leur signification.

d) La relation traditionnelle : dans la contagion affective, un mot d'abord intellectuel et de nature générale est traditionnellement et étroitement lié à une situation chargée d'émotivité par la conscience populaire. Ce terme a subi la contagion d'une situation traumatique, exaltante ou extraordinaire par quelque côté. Tels sont les mots mort, pendaison, tuer, assassin, naissance, sexe, amour, haine, vice, vertu, infini, bombe atomique, bombe à hydrogène, mensonge, avarice et leurs équivalents anglais. Hiroshima, Nagasaki, Gestapo, Nuremberg joignent une relation géographique et temporelle à la contagion affective, ce qui leur fournit plusieurs couches affectives. Employés dans des contextes intellectuels, ces mots peuvent reprendre leur neutralité expressive, mais, insérés dans des situations, des conversations ou des contextes le moindrement chargés, ils prennent pour leur propre compte l'émotivité dont ils sont entourés et revalorisent ainsi la qualité ou la quantité latente dans le concept :

The high brick wall in front of the school gave it the look of a prison 36 .

How he felt useless : he was a criminal and ought only to talk to criminals... ${ }^{37}$

"I hate you. I wish you was dead", Mrs. Carey gasped 38 .

He made up his mind now that nothing Josiah Graves said would induce him to remove the candlesticks from the altar, and he muttered Bismark to himself once or twice irritably 39 .

Qu'importait si, accusé de meurtre, il était exécuté pour n'avoir pas pleuré à l'enterrement de sa mère 40 ?

De tels exemples abondent. C'est souvent ce qui explique l'émotivité d'une langue blanche : à témoin le dernier exemple.

2. Relation linguistique : il s'agit cette fois d'un déplacement sémantique qui s'ajoute à un déplacement de situation : c'est l'équạtion entre deux réalités dont l'une est absente de la situation et l'autre présente. La ressemblance partielle entre elles permettant leur équation force le mot représentant la réalité absente à faire un circuit sémantique. "Cet homme est un lion. "Homme constitue la réalité

34. James Joyce, Dubliners, New York, The Viking Press, 1958, p. 134.

35. André Gide, la Symphonie pastorale, Paris, Le Livre de Poche, 1964, p. 19.

36. Somerset W. Maughan, op. cit., p. 17.

37. Graham Greene, op. cit., p. 124 .

38. Somerset W. Maughan, op. cit., p. 16.

39. Somerset W. Maughan, op. cit., p. 14

40. Albert Camus, l'Étranger, Paris, Le Livre de Poche, 1968, p. 177. 
présente, lion la réalité absente. C'est la mise en gros plan de lion par son équation avec homme qui fait ressortir un seul aspect de lion, à savoir la force ou la bravoure (portée à un haut degré). La force, étant une réalité dynamique, exprime l'intensité, la nouvelle intensité de lion. La métaphore revalorise les éléments expressifs d'un terme. Dans l'exemple qui nous occupe, l'intensité latente dans la signification de ce mot est revalorisée par le déplacement sémantique, si temporaire soit-il. Il va sans dire que ce terme, quoique toujours de nature générale, prend une nouvelle expressivité. Il faut ajouter que la métaphore EN sor n'éveille qu'une affectivité accidentelle, étant donné que cette relation entre deux réalités et la situation n'est mise en évidence que dans un contexte optionnel et renouvelable.

Lorsque la métaphore se trouve dans une situation ou un contexte n'admettant aucune ambiguïté, l'esprit fait un raccourci, met à l'arrière-plan la réalité présente dans la situation. Dans notre exemple, homme disparaît et l'on dit : c'est un lion. L'intensité en regagne d'autant. On note en passant les couches affectives que pourraient ajouter l'intonation, le ton, le geste, à de tels énoncés. Ceux-ci ne font pourtant pas l'objet de cette analyse. Voici des exemples semblables :

Rivière décrocha l'écouteur, reçut le bourdonnement du monde 41.

Minne décroche enfin son regard de la fenêtre ouverte... ${ }^{42}$

He was scared, and yet a curious pride bubbled in his throat... ${ }^{43}$

«Ce bruit est un bourdonnement. » « Cesser de regarder la fenêtre » c'est « décrocher le regard... » «Pride made itself felt $»=$ pride bubbled. Chaque métaphore est le résultat d'une équation. Les réalités présentes dans la situation - bruit, cesser, make oneself felt - sont implicites, tandis que les réalités absentes de cette situation particulière sont explicitées. Bourdonner, c'est le propre d'un insecte, non du monde; on décroche un récepteur, non un regard; water bubbles, non pride. On a donc saisi ces réalités et on les a insérées dans une situation étrangère à la leur: Ce faisant, on oblige l'esprit à opérer une espèce de crochet sémantique : il doit saisir la signification propre du mot employé dans une situation qui lui est propre (la mouche bourdonne) avant de comprendre le déplacement de situation par ressemblance analogique (le monde : nouvelle situation de bourdonner). C'est cette ressemblance qui devient le nouveau contenu sémantique de la métaphore. L'alliance entre le nouveau signifié et le mot lui-même se dissout dès que cesse le déplacement de situation. C'est dire que la métaphore reprend son sens propre dans un nouveau contexte non métaphorique, si la situation lui convient. C'est ce qui explique la métaphore éteinte : «la nuit tombe ». Ici on oublie la situation propre au mot tomber (un corps, un objet tombent), et l'on en vient à sentir que la nouvelle situation (la nuit) est normale. L'esprit cesse de faire un circuit sémantique ; la nouvelle signification s'attache au mot.

Dans certains cas, tels que « la faim le tenaille », « il est tenaillé de remords », la métaphore est encore vivante, même si la réalité et la situation normale sont sorties de nos mœurs, car la conscience linguistique a encore conscience de sa situation propre, probablement en raison des musées, des romans, des écrits histo-

41. Antoine de Saint-Exupéry, op. cit., p. 76.

42. Colette, op. cit., p. 32.

43. Graham Greene, op. cit., p. 43. 
riques, des tortures infligées pendant les deux guerres mondiales. II faut toutefois attirer à l'attention le fait suivant : la métaphore éteinte du genre \& la nuit tombe », ne peut faire l'objet de notre étude en tant que métaphore morte, car la stylistique doit rester à l'intérieur d'une période donnée et ce n'est que dans une perspective historique qu'une métaphore est dite éteinte. Nous tombons autrement dans le domaine de la sémantique diachronique.

La comparaison est essentiellement une métaphore mais à cette différence près, à savoir que le déplacement de situation est explicité formellement par une particule du genre comme, de, semblable à, etc.

... voilà le clocher bleu qui monte au-dessus des bois comme une fleur et, au bout d'un petit moment, voilà sa campane qui sonne l'Angélus AVEc la voix d'une clochasse de bouc ${ }^{44}$.

Le Panturle est un homme énorme. ON DIRAIT un morceau de bois qui marche 45 .

He accepted a piece of half-raw meat and gnawed it LIKE a wolf 46 .

... spots of blurred sunlight slid over their bodies or moved LIKE bright, winged things in the shade ${ }^{47}$.

La fleur n'a pas la manière d'être du clocher, et la voix d'une clochasse de bouc n'est pas dans la situation, par rapport à ce qui l'entoure, d'une campane qui sonne l'Angélus. L'explicitation formelle du déplacement de situation par les particules COMME, ON DIRAIT, etc., atténue le déplacement sémantique et diminue d'autant l'expressivité.

Le dédoublement, cas où le terme est à la fois métaphorique et au sens propre, comme dans l'exemple suivant, peut parfois doter la même réalité à la fois d'une présence et d'une absence :

La lune pose son doigt blanc sur ses paupières.

C'est une autre façon de dire que l'homme dort sous la lune. La lune est présente comme phénomène naturel, mais la lune est absente comme personne. Lune est en même temps direct (sens propre) et métaphorique (ce mot est personnifié) : donc dédoublé. Poser, doigt, blanc, sont absents et donc simplement métaphoriques, tandis que paupière n'est qu'une simple présence (sens propre) dans l'actualité fictive. La présence et à la fois l'absence de la personne (lune) constituent une espèce de contradiction ressentie par l'affectivité, d'autant plus que cette personnification touche au merveilleux. L'expressivité en regagne. De plus, une telle phrase est éminemment littéraire. Elle comporte donc une relation sociale : donc une nouvelle couche affective ${ }^{48}$.

Dans les cas suivants le dédoublement est sur vent, soleil, vie, sleep, stars, fire, trees:

Enfin, on abordera le plateau, l'étendue toute rabotée par la grande varlope de ce vent 49 . Le vent hurle derrière les nuages 50 .

44. Jean Giono, op. cit., p. 5.

45. Jean Giono, op. cit., p. 16.

46. William Golding, Lord of the Flies, New York, Capricorn Books, 1954, p. 67.

47. William Golding, op. cit., p. 12.

48. Sur toutes ces questions : le mot-image, la métaphore et la comparaison, voir Yvonne Saint-Pierre Farina, les Cadres expressifs comparés de l'anglais et du français (à paraître à Paris, chez Didier, "Bibliothèque de stylistique comparée ").

49. Jean Giono, op. cit., p. 8.

50. Jean Giono, op. cit., p. 11 . 
Le soleil nageait tout blond autour de la pièce de bois 51 .

Cette vie est un hôpital où chaque malade est possédé du désir de changer de lit 52 .

... he did not know how quickly sleep came and hurled him down a dark interior slope 53 .

Again the stars spilled about the sky 54.

... the fire laid hold on the forest and began to gnaw 55 .

... the trees of the forest sighed, then roared 56 .

Il existe d'autres types de dédoublement que nous reprendrons en temps et lieu. Le dédoublement est d'une grande fréquence. Son expressivité varie d'un contexte à l'autre, et son rôle, dans la formation des images syntaxiques, est éminemment dynamique et concentré.

\section{La modalité concrète}

Le concret et l'affectivité constituent les deux éléments de l'expressivité. Le concret relève les notations précises des sens : le visuel, l'auditif, le tactile, le gustatif, l'olfactif, etc. Il côtoie le réel pour nous en donner une image détaillée. Les mots à dominance concrète sont toujours des mots-images. Ceux-ci se placent sur le plan du réel, comme le disent Vinay et Darbelnet. En voici des exemples :

visuel : Tout ce qui couvrait une vie humaine déjà scintillait ${ }^{50}$.

tactile : Panturle embrasse le tronc gluant...58

auditif : Voici la sonnerie redoutée, les sept coups stridents qui vibrent...59

olfactif : ... où l'atmosphère est parfumée par les fruits, par les feuilles et par la peau humaine 60 .

gustatif : Irène Chaulieu mordille une rose et réfléchit...61

tensoriel : En es-tu donc venue à ce point d'engourdissement que tu ne te plaises que dans ton mal 62 ?

auditif : Then Eric sniggered 63 .

visuel : Ralph spurted again and missed 64.

auditif et visuel : ... there came a splashing of water...65

auditif : Hermione laughed in her short, grunting fashion 66.

tactile et visuel : He mopped his face 67.

tactile, tensoriel et visuel : ... that plump incarnation of turpitude writhing in the heather of his feet 68 .

51. Jean Giono, op. cit., p. 19.

52. Charles Baudelaire, op. cit., p. 137.

53. William Golding, op. cit., p. 176.

54. William Golding, op. cit., p. 174.

55. William Golding, op. cit., p. 39.

56. William Golding, op. cit., p. 91.

57. Antoine de Saint-Exupéry, op. cit., p. 22 ss.

58. Jean Giono, op. cit., p. 90.

59. Colette, op. cit., p. 107.

60. Charles Baudelaire, op. cit., p. 51.

61. Colette, op. cit., p. 177.

62. Charles Baudelaire, op. cit., p. 138.

63. William Golding, op. cit., p. 90.

64. William Golding, op. cit., p. 136.

65. D. H. Laurence, Women in Love, New York, The Viking Press, 1968, p. 177.

66. D. H. Laurence, op. cit., p. 292.

67. Aldous Huxley, Brave New World, New York, Bantam Books, 1968, p. 172.

68. Aldous Huxley, op. cit., p. 176. 
On a conscience d'une précision parce que, à côté d'eux, des mots généraux et conceptualisants rappellent le même concret mais d'une façon neutre; incolore et universelle : briller (scintillait), humide (gluant), désagréable (strident), sonner (vibrent), remplie (parfumée), insensibilité (engourdissement), etc. Comme le concept des mots-images est déjà représenté dans la langue par des mots incolores et neutres, il faut croire que le rôle spécial de ces images lexicales est de présenter des modalités détaillées et précises.

\section{Le cumul expressif}

L'intensité, la qualité, la relation et la modalité concrète peuvent, chacune, s'isoler dans un terme ou encore se cumuler.

... monkeys crashed invisibly among the trees with an effect of clumsiness and recklessness, and what were probably snakes, hissed away LIKE match-flames tbrough the grass 69 .

Ici, seuls les mots qui sont en italique sont vraiment expressifs. Les autres sont neutres ou à peu près. On note que ces termes cumulent les couches expressives :

crashed : intensité : augmentative ; qualité : légère nuance péjorative ; modalité : auditive (dominante).

clumsiness : intensité : légère nuance intensive, augmentative; qualité : péjorative (dominante) ; modalité : concret imprécis, tactile, visuel et auditif.

recklessness : intensité : légère nuance intensive, augmentative ; qualité : péjorative (dominante) ; modalité : tactile, concret imprécis.

snakes : relation : contagion affective ; relation sociale : familière ; modalité : auditive, précise en raison de l'onomatopée.

hissed away : intensité : augmentative (dominante); qualité : péjorative (dominante); relation : contagion affective; relation linguistique : comparaison; modalité : auditive (hissed) ; visuelle (away).

match-flames : modalite : visuelle; relation : linguistique, comparaison.

Analysons un autre exemple :

Dans quelle abominable nuit je plonge 70 !

Cẹla signifie : je deviens très triste.

Dans : relation : linguistique, métaphorique.

quelle : fait de grammaire expressive : intensité augmentative.

abominable : intensité : augmentative; qualité : péjorative; relation : linguistique; dédoublement (métaphorique : adjoint de nuit; s'applique en même temps à triste). nuit : relation : sociale. Niveau littéraire, usage; relation : linguistique. Métaphore, revalorise les éléments intensifs et péjoratifs latents dans le mot.

plonge : intensité : augmentative; relation : sociale. Niveau littéraire, usage; relation : linguistique. Métaphore ; concret : visuel et tensoriel.

L'on s'aperçoit que le schéma de l'expressivité, présenté dans notre exposé, suggère un début de méthode pour l'analyse expressive, que celle-ci soit en stylistique anglaise, en stylistique française ou en stylistique comparée. On note, en plus, que le cumul expressif peut se trouver dans le même mot. Le cumul est alors vertical, car les couches expressives se superposent et deviennent des composantes

69. Graham Greene, op. cit., p. 149.

70. André Gide, op. cit., p. 146. 
sémantiques, saisies toutes à la fois par la conscience linguistique de l'homme moyen, bien qu'elles puissent très bien ne pas trouver leur place dans le dictionnaire. On remarque aussi que les segments de phrase juxtaposent dans le temps ces couches expressives. Le cumul expressif est alors horizontal.

En résumé, l'expressivité tourne sur deux axes : l'affectivité et le concret. L'affectivité saisit la qualité, l'intensité et la relation, tandis que le concret exprime la modalité particularisánte. Ces éléments se trouvent isolés ou ensemble. Ils peuvent se cumuler dans le même terme et se juxtaposer dans un énoncé. La métaphore et le mot-image sont pris dans cet engrenage et suivent les mêmes pentes.

Yvonne SaInt-Pierre Farina 